\title{
SYNTHESIS OF DEVELOPMENT SCENARIOS OF COMPLEX SYSTEMS
}

\author{
Vladimir V. Kul'ba and Dmitry A. Kononov \\ Trapeznikov Institute of Control Sciences \\ of Russian Academy of Sciences \\ 65, Profsoyuznaya str. Moscow, Russia, 117806, \\ E-mail: kulba@ipu.rssi.ru
}

\author{
Galina Merkuryeva \\ Riga Technical University \\ 1 Kalku Street \\ Riga, Latvia, LV-1658 \\ E-mail: gm@itl.rtu.lv
}

\section{KEYWORDS}

Scenario, control, safe development.

\begin{abstract}
This paper presents new theoretical results of the methodology elaboration for the scenario-based research of complex systems. Basic techniques and tasks of the scenario-based research of social economic systems are formulated. Basic concepts of behaviour scenario, as well as of its elements and their characteristics are defined. Tasks of optimal synthesis of synergetic and attractive scenarios of social economic system development are determined.
\end{abstract}

\section{INTRODUCTION}

According to the suggested formalised methodology of the scenario-based research of social economic systems (SES), the process of modelling, planning and control implementation is performed on the basis of integration of system logical, structure-social and scenario-based approaches (Kononov et al. 2005).

The system logical approach assumes constructing and studying objects of research from the position of systems analysis whose underlying concept is a formal system object. This allows one to study objects and processes from the viewpoint of formal logical and mathematical methods.

The structure-social approach assumes constructing and studying social economic objects of control on the basis of definition and structuralization of human activity types. This enables studying objects and processes in social systems at different strata of society's social structure and distinguishing main social objects and social structures as well as describing different social processes on that basis.

A combination of system logical and structure-social approaches makes it possible to study human activity as the main motive reason of social economic system development, considering it as the structured spectrum of formalised processes of state changes of the social economic objects and subjects of action.

The scenario-based approach supposes investigation of the processes occurring in social economic systems on the basis of construction and investigation of behaviour scenarios (synergetic scenarios) of social subjects of action and scenarios of control (attractive scenarios) of social objects.

A combination of system logical, structure-social and scenario-based approaches allows scenario-based research on the basis of construction of the scenario system representing both general and specific characteristics of SES, its elements, the discovered relationships among them and indicated features of those relationships. An effectively constructed scenario system helps solve multi-aspect problems, perform scenario-based analysis and synthesise scenarios of rational behaviour of different social subjects of action, which makes it possible to pass on to the construction of a safety ensuring system of the given object, structure or process of SES.

In (Kononov 2007), impulse synergetic and attractive scenarios of direct control of SES development are discussed. It is found that in principle there exist only six possible scenarios of such development. This paper continues describing results of mathematical investigation of SES behaviour model constructed using the language of operator directed graphs (Roberts 1976).

\section{SYNTHESIS OF SES DEVELOPMENT SCENARIOS}

The typology of applied systems described in (Kul'ba et al. 2004) enables SES structuralization and studying its subsystems using methods of the scenario-based analysis. Examples of SES development modelling give evidence that even at the classification level a number of their characteristic structural peculiarities can be distinguished. They can be grouped by features which determine the unique properties and relationships among the macroindicators of the revealed classification group. Using formal operations in the considered scenario spaces, it is then possible to make an effective analysis and accomplish the synthesis of optimal development scenarios and thus construct a transformation scenario.

The scenario $\mathfrak{R}$ as an object of research is a complex hierarchically constructed structure (Kononov 2001). Let us distinguish its basic elements:

- General elements of a model: a metaset $\mathbf{M}=\left(M_{O} ; M_{E} ; M_{D}, M_{M O} ; M_{M E} ; \hat{A}\right)$, sets of internal states $Y \subseteq E^{m}$ and environmental states of SES $X \subseteq E^{n}$, expanded phase space $X \times Y=Z \subseteq E^{n+m}$ and 
its expert significant partition
$\Xi=\left\{\left\{Z^{(\alpha)}\right\}, A^{(e)}\right\}$

- Spatial information elements: trajectory scale ZT, event scale $\Re T$, an event significant to expert (ESE) $\mathfrak{J}_{e v}^{\alpha}(\mathbf{z}, t)$, depth $N \in \mathfrak{R} T$, horizon $t_{N}=T \in Z T$, conditional decision $\varsigma\left(t_{i}\right)=\left(\vartheta\left(t_{i}\right), \gamma\left(t_{i}\right)\right)$ at time instant $t_{i} \in Z T$, including also controllable operated factors (the CO factors) $\vartheta \in M_{0} \subseteq E^{d}$, uncontrollable factors $\gamma \in \Gamma \subseteq E^{\gamma}$ and a set of conditional decisions $S^{(c s)}=M_{0} \times \Gamma$.

The scenario $\mathfrak{R}$ with the initial event ESE $\mathfrak{J}_{e v}^{(\alpha(0))}\left(t_{0}\right)=\left\{Z^{(\alpha(0))}, \mathbf{z}\left(t_{0}\right), t_{0}\right\}$, is constructed with regard to trajectory scales $Z T$ and event scales $\mathfrak{R} T$, using these rules:

$$
\mathfrak{R}=\mathfrak{R}\left\{\left(I\left(t_{i}\right), t_{i}\right)\right\},
$$

where $t_{i} \in \hat{A}$ for $i=0,1, \ldots, N ; t_{0}=0$,

$$
I\left(t_{i}\right)=\left(S\left(t_{i}\right), M^{(Q I H)}\left(t_{i}\right)\right) \text {, }
$$

where $t_{i} \in \widehat{A}$ for $i=0,1, \ldots, N ; t_{0}=0$.

Conditions $I\left(t_{i}\right)$ at $t_{i} \in Z T \subseteq R$ are characterised by situation $S\left(t_{i}\right)$ and a quasi-information hypothesis $(\mathrm{QIH}), M^{(Q I H)}\left(t_{i}\right)$.

A situation is a set of events which occurred before time instant $t$ :

$S(t)=\left\{\mathfrak{J}^{(i)}\left(\mathbf{z}^{(i)}\left(t_{i}\right), t_{i}\right) 0 \leq t_{i} \leq t, i=0,1, \ldots, s ; t_{0}=0\right\}$ (3)

whereas the expected event is the expected realisation of $\mathbf{z}(t) \in Z$ factors of the expanded phase trajectory in the elementary partition (EP) $Z^{\alpha} \in \Xi$ at time instant $t$ :

$$
\mathfrak{I}_{e v}^{\alpha}(t)=\left\{Z^{\alpha}, \mathbf{z}(t), t\right\} .
$$

The occurrence of the next in turn ESE $\mathfrak{J}_{e v}^{\left(\alpha_{i+1}\right)}\left(t_{i+1}\right)$ of scenario $\mathfrak{R}$ at time instant $t_{i+1}$ with regard to scale $Z T$, or at time instant $i+1$ with regard to the discrete scale $\mathfrak{R} T$, is determined by scenario forming elements: situation $S\left(t_{i}\right)$, QIH $M^{(Q I H)}\left(t_{i}\right)$ and scenario formation strategy $\breve{C}\left(t_{i}\right)$ at time instant $t_{i}$ as a ternary relationship:

$$
\mathfrak{J}_{e v}^{\left(\alpha_{i+1}\right)}\left(t_{i+1}\right)=\left(S\left(t_{i}\right), M^{(Q I H)}\left(t_{i}\right), \breve{C}\left(t_{i}\right)\right) .
$$

Each of the above mentioned elements characterises the process of its construction in a certain aspect:

$S\left(t_{i}\right)$ - situation described by a set of most important SES parameters; determines phase conditions under which the researcher has to make a decision;

$M^{(Q I H)}\left(t_{i}\right)$ - quasi-information hypothesis; it represents the researcher's knowledge structure and actually represents a model of the uncertainty taken into account (Kul'ba et al. 2004);

$\breve{C}\left(t_{i}\right)$ - scenario forming strategy; it determines those scenario continuation techniques which the operating party is able to afford.

In general, scenarios of three basic types can be determined and constructed:

- Synergetic scenarios, which model behavioural aspects of the system under consideration and describe the development spectrum provided that there are no control influences on SES functioning processes;

- Direct control scenarios, which model the development spectrum in connection with the chosen set of arrangements realising the direct control task;

- Attractive scenarios, which characterise the behaviour of a SES in accordance with the desirable - reasonable from the viewpoint of the decision maker's aim - control influences, i.e. realising the inverse control task. Control is determined as a result of realisation of the current situations which, in particular, occur under the influence of external events and of decision maker's decisions. Within that scheme, a scenario can be synthesised as an instrument of formal analysis of alternative situation development variants at the specified aims in the presence of uncertainty.

The occurrence of the next in turn ESE $\mathfrak{J}_{e v}^{\left(\alpha_{i+1}\right)}\left(t_{i+1}\right)$ of the synergetic scenario is determined by general elements of the model and expert scenario forming elements according to rule (5). The occurrence of the next in turn ESE of the attractive scenario additionally requires that the rules of operation with spatial information components aimed at making control conditional decision must be clarified according to the following scheme:

- rule $\mathbf{A}^{(A)}\left(t_{i}\right) \subseteq \hat{A}$ for evaluating the effectiveness of the attractive strategy $\breve{C}^{(A)}\left(t_{i}\right)$, which enables finding conditional decision $\varsigma\left(t_{i}\right)=\left(\vartheta\left(t_{i}\right), \gamma\left(t_{i}\right)\right) \in S^{(c s)}$;

— rule $\Lambda^{-1}\left(\vartheta\left(t_{i}\right), t_{i}\right)$ for determining elements of the $\mathrm{CO}$ factor;

- rule $\mathbf{A}^{(\text {fixm })}\left(t_{i}\right) \subseteq \widehat{A}$ for fixing the time of the next in turn ESE $\mathfrak{J}_{e v}^{\left(\alpha_{i+1}\right)}\left(t_{i+1}\right)$.

Formally, a strategy is defined as a ternary relationship:

$$
\breve{C}^{(A)}\left(t_{i}\right)=\left(\mathbf{A}^{(A)}\left(t_{i}\right), \Lambda^{-1}\left(\vartheta\left(t_{i}\right), t_{i}\right), \mathbf{A}^{(f i x m)}\left(t_{i}\right)\right) .
$$

As soon as at time instant $t \in Z T$ a QIH is fixed in the form of the structured subset $M^{(Q I H)}(t) \subseteq S^{(c s)}=Z^{0}$ of the expanded phase space $Z$, the next in turn ESE can be determined by indicating the $\mathrm{CO}$ factor $\vartheta=\Lambda(\mathbf{u}, \mathbf{p}, \Delta)$ in accordance with the chosen strategy $\breve{C}$ for scenario $\mathfrak{R}$ formation. $\breve{C}$ is constructed using rules $A^{(s)} \subseteq \widehat{A}$. Each point $\mathbf{z}=(\mathbf{x}, \mathbf{y}) \in Z$ along with the 
fixed rules of trajectory $\mathbf{A}^{(f i x m)}\left(t_{i}\right) \subseteq \widehat{A}$ discretisation at each time instant $t_{i} \in Z T \quad i=0,1, \ldots$ (or on a certain horizon of scenario $T \in Z T$ ) and elements of metaset $\mathbf{M}$ determine object $\mathfrak{R}\left(\mathbf{z}, I\left(t_{i}\right), t_{i}\right)$ behaviour scenario, outgoing from point $\mathbf{z}$, as a sequence of QIH, scenario formation strategies, rules for choosing them and expected events corresponding to them. The representation of scenario $\mathfrak{R}$ as a sequence of ESE and QIH, that is conditions 0 , is oriented towards the decision maker or external -with regard to the process of problem analysisuser. In contrast to 0 , a detailed technology for obtaining a sequence of that kind in the form of a set of strategies and rules of choice 0 is intended for the expert dealing with the statement and solving of the problem under study. The problem of the formalised synthesis of SES functioning and development scenarios is a topical task. The underlying idea of the suggested process of complex system development modelling is the elaboration of scenarios of their behaviour for the specified target analytical purposes, which will result in the synthesis of the scenario with the specified properties, including an optimal scenario.

The optimality of a scenario can be considered from two viewpoints.

The first one consists of the verbal statement of the problem of scenario generation as a choice of the most rational (optimal) expert description of problem situations fixing the most essential properties of the considered process of complex system behaviour. The optimality property is underlying for the scenario to be formed, as it distinguishes that description technique from related concepts: a forecast, a plan and a trajectory. This is the choice of the next in turn expert meaningful event - but not of all possible ways of development or goal achievement in terms of the expanded phase space- which determines the scenario to be formed. The indication of the appropriate sequence of ESE at the given assumptions about the course of object development depends on the goals of research (the synergetic approach) or on control (the attractive approach). Thus, we should speak about the optimality of scenario formation. The principles and a sequence of optimal scenario formation according to the concept are described in (Kononov 2001).

An alternative concept of scenario optimality from the viewpoint of the scenario-based research is formulated as choosing an optimal scenario out of the specified scenario set in the scenario space under consideration. Such scenarios can be obtained irrespective of the researcher; his task is to determine "the best" of them.

The multiplicity of system behaviour scenarios becomes apparent in qualitatively different sides of the system under study, which are fixed to determine a strategy in the ascertained quasi-information hypothesis of the operating party in synthesising the scenario. Within the formalized approach, the manysideness of study object is represented in the diversity of its characteristics and properties.

By a characteristic we understand the qualitative side of study object formalised in the form of a formal model. A numeric function $f$ corresponding to the chosen scale is called a numeric characteristic- or measurement indicator- of an object; a Boolean characteristic of an object is a model of object's property; other models of the characteristics are also possible.

A set of characteristics $\mathbf{f}^{(e)}$ of element $e(\mathfrak{R})$ of scenario $\mathfrak{R}$ as well as other components of the scenario system can serve as an initial set of synthesis characteristics.

Metric characteristics of elements of scenario determine its properties as an element of the expanded phase space $Z \subseteq E^{n+m}$, of the scenario space $Z^{(s c)}$, as well as of metric space $\breve{\mathrm{M}}\left(Z, \rho_{Z}\right)$ and normalised space $\operatorname{Nor}\left(Z,\|\|_{Z}\right)$ constructed on their basis.

A number of characteristics of general elements of the model of the scenario system are described in (Kul'ba et al. 2004).

Within the ESP-model (Kul'ba et al. 2004), an ESE $\mathfrak{\Im}_{e v}^{\alpha}(t)$ can be considered to be an element of space

$$
\begin{gathered}
\mathfrak{I}_{e v} Z T=Z \times Z \times Z T \subseteq E^{2(n+m)+1} \\
\left(Z^{\alpha} \subseteq Z, \mathbf{z}(t) \in Z, t \in Z T\right),
\end{gathered}
$$

which will be called the space of expert significant events (SESE).

To calculate characteristics of ESP and ESE, it is necessary to define a metric space $\breve{M}\left(M \mathfrak{J}_{e v}, \rho_{e v}\right)$, the distance in which is determined by metric $\rho_{e v}$ (for instance, introduce metric $\rho_{Z}=\rho\left(\mathbf{z}^{(1)}, \mathbf{z}^{(2)}\right)$ in the expanded phase space $Z$ )

$$
M \mathfrak{I}_{e v} \subseteq \mathfrak{J}_{e v} Z T .
$$

Examples of synergetic and attractive characteristics of scenarios in the metric space 0 are given in (Kul'ba et al. 2004). While forming a scenario, different strategies can be used, whose application depends on the circumstances of searching for the next in turn ESE: the situation, the model of accounted uncertainty (QIH), rules of taking it into account etc. It is proposed to consider the search for an effective strategy in two stages: accounting for the uncertainty and optimisation. A number of characteristics of uncertainty $H^{(I)}$ accounting techniques and a strategy of functional optimisation $H^{(O)}(\vartheta)$, including optimism measure, variativity measure etc., are defined in (Kul'ba et al. 2004, Kononov 2001). As a more complicated example of QIH characteristic, the structure related to the reflexive model of uncertainty 
constructed in (Novikov and Chartishvili 2003), can be considered.

A set of characteristics

$$
\mathbf{f}(\mathfrak{R})=\left\{f_{\tau}(\mathfrak{R}) \tau \in \Sigma\right\}
$$

of scenario $\mathfrak{R}$ is as a rule a convolution

$$
f_{\tau}(\mathfrak{R})=\Theta_{\tau}\left(\mathbf{f}^{(e)}(\mathfrak{R})\right)
$$

of partial characteristics $\mathbf{f}^{(e)}$ of elements $\mathbf{e}(\mathfrak{R})$ constituting scenario $\mathfrak{R}$. Most frequently, the permissibility and/or desirability of the formed scenario are indicated as a set of its properties. In different applications, these properties are distinguished:

- achievement of the given observability extent of the object and its surrounding;

- achievement of the specified completeness of object behaviour description;

- achievability scenario;

- safety scenario;

- scenario realisticity etc. (Kul'ba et al. 2004).

Due to that, determination of transformation 0 is an independent task.

Assume direction $\mathbf{a} \in Z$ and a positive number $\delta$ are set. Let us give some spatial characteristics of scenarios: -current remoteness of scenario $\mathfrak{R}\left(t_{0}, T\right)$ from vector $\mathbf{a}$

$$
d_{E}^{(t)}\left(\mathfrak{R}\left(t_{0}, T\right), \mathbf{a}\right)=\rho_{E}(\mathbf{z}(t), \mathbf{a})=\|\mathbf{z}(t)-\mathbf{a}\|_{E} ;
$$

--current angular remoteness of scenario $\mathfrak{R}\left(t_{0}, T\right)$ from vector $\mathbf{a}$

$$
d_{a}^{(t)}\left(\mathfrak{R}\left(t_{0}, T\right), \mathbf{a}\right)=\rho_{a}(\mathbf{z}(t), \mathbf{a})=\left\|\frac{\mathbf{z}(t)}{\|\mathbf{z}(t)\|_{E}}-\frac{\mathbf{a}}{\|\mathbf{a}\|_{E}}\right\|_{E} ;
$$

-minimal remoteness of scenario $\mathfrak{R}\left(t_{0}, T\right)$ from vector a on the horizon $T$

$$
d_{E}^{\min }\left(\mathfrak{R}\left(t_{0}, T\right), \mathbf{a}\right)=\min _{\mathbf{z} \in \mathfrak{R}\left(t_{0}, T\right)}\|\mathbf{z}-\mathbf{a}\|_{E}=\min _{t \in t_{0}, T} d_{E}^{(t)}\left(\mathfrak{R}\left(t_{0}, T\right), \mathbf{a}\right) ;
$$

-minimal angular remoteness of scenario $\mathfrak{R}\left(t_{0}, T\right)$ from vector a on the horizon $T$

$d_{a}^{\min }\left(\mathfrak{R}\left(t_{0}, T\right), \mathbf{a}\right)=\min _{\mathbf{z} \in \mathfrak{R}\left(t_{0}, T\right)} \rho_{a}(\mathbf{z}, \mathbf{a})=\min _{t \in t_{0}, T} d_{a}^{(t)}\left(\mathfrak{R}\left(t_{0}, T\right), \mathbf{a}\right) ;$ -maximal remoteness of scenario $\mathfrak{R}\left(t_{0}, T\right)$ from vector a on the horizon $T$

$$
d_{E}^{\max }\left(\mathfrak{R}\left(t_{0}, T\right), \mathbf{a}\right)=\max _{\mathbf{z} \in \mathfrak{R}\left(t_{0}, T\right.}\|\mathbf{z}-\mathbf{a}\|_{E}=\max _{t \in t_{0}, T} d_{E}^{(t)}\left(\mathfrak{R}\left(t_{0}, T\right), \mathbf{a}\right) ;
$$

-maximal angular remoteness of scenario $\mathfrak{R}\left(t_{0}, T\right)$ from vector a on the horizon $T$

$$
d_{a}^{\max }\left(\mathfrak{R}\left(t_{0}, T\right), \mathbf{a}\right)=\max _{\mathbf{z} \in \mathfrak{R}\left(t_{0}, T\right)} \rho_{a}(\mathbf{z}, \mathbf{a})=\max _{t \in t_{0}, T} d_{a}^{(t)}\left(\mathfrak{R}\left(t_{0}, T\right), \mathbf{a}\right)
$$

-a set of periods when scenario $\mathfrak{R}\left(t_{0}, T\right)$ exceeds the

bounds of $\delta$-vicinity of vector $\mathbf{a}$

$$
T_{E}^{(t, \text { out })}\left(\mathfrak{R}\left(t_{0}, T\right), \mathbf{a}, \delta\right)=\underset{t_{0} \leq t \leq T}{\operatorname{Arg}}\left(d_{E}^{(t)}\left(\mathfrak{R}\left(t_{0}, T\right), \mathbf{a}\right) \geq \delta\right) ;
$$

-the first moment when scenario $\mathfrak{R}\left(t_{0}, T\right)$ exceeds the bounds of $\delta$-vicinity of vector $\mathbf{a}$

$$
T_{E}^{(\min , \text { out })}\left(\mathfrak{R}\left(t_{0}, T\right), \mathbf{a}, \delta\right)=\min _{t_{0} \leq t \leq T}\left(T_{E}^{(t, \text { out })}\left(\mathfrak{R}\left(t_{0}, T\right), \mathbf{a}, \delta\right)\right) ;
$$

-the last moment when scenario $\mathfrak{R}\left(t_{0}, T\right)$ exceeds the bounds of $\delta$-vicinity of vector $\mathbf{a}$

$$
T_{E}^{(\text {max,out })}\left(\mathfrak{R}\left(t_{0}, T\right), \mathbf{a}, \delta\right)=\max _{t_{0} \leq t \leq T}\left(T_{E}^{(t, \text { out })}\left(\mathfrak{R}\left(t_{0}, T\right), \mathbf{a}, \delta\right)\right) ;
$$

-a set of periods of scenario $\mathfrak{R}\left(t_{0}, T\right)$ staying in $\delta$ vicinity of vector $\mathbf{a}$

$$
T_{E}^{(t, i n)}\left(\mathfrak{R}\left(t_{0}, T\right), \mathbf{a}, \delta\right)=\underset{t_{0} \leq t \leq T}{\operatorname{Arg}}\left(d_{E}^{(t)}\left(\mathfrak{R}\left(t_{0}, T\right), \mathbf{a}\right) \leq \delta\right) ;
$$

- the first moment of scenario $\Re\left(t_{0}, T\right)$ falling in $\delta$ vicinity of vector $\mathbf{a}$

$$
T_{E}^{(\min , i n)}\left(\mathfrak{R}\left(t_{0}, T\right), \mathbf{a}, \delta\right)=\min _{t_{0} \leq t \leq T}\left(T_{E}^{(t, i n)}\left(\mathfrak{R}\left(t_{0}, T\right), \mathbf{a}, \delta\right)\right) ;
$$

- the last moment of scenario $\mathfrak{R}\left(t_{0}, T\right)$ staying in $\delta$ vicinity of vector $\mathbf{a}$

$T_{E}^{(\text {max }, i n)}\left(\mathfrak{R}\left(t_{0}, T\right), \mathbf{a}, \delta\right)=\max _{t_{0} \leq t \leq T}\left(T_{E}^{(t, i n)}\left(\mathfrak{R}\left(t_{0}, T\right), \mathbf{a}, \delta\right)\right) ;$

- current velocity of scenario $\mathfrak{R}\left(t_{0}, T\right)$ in the direction of vector $\mathbf{a}$

$$
v_{E}^{(t)}\left(\mathfrak{R}\left(t_{0}, T\right), \mathbf{a}\right)=d_{E}^{(t)}\left(\mathfrak{R}\left(t_{0}, T\right), \mathbf{a}\right) / t ;
$$

- current angular velocity of scenario $\mathfrak{R}\left(t_{0}, T\right)$ with regard to vector $\mathbf{a}$

$$
v_{a}^{(t)}\left(\mathfrak{R}\left(t_{0}, T\right), \mathbf{a}\right)=d_{a}^{(t)}\left(\mathfrak{R}\left(t_{0}, T\right), \mathbf{a}\right) / t ;
$$

- minimal velocity of scenario $\mathfrak{R}\left(t_{0}, T\right)$ in the direction of vector $\mathbf{a}$

$$
v_{E}^{(\min )}\left(\mathfrak{R}\left(t_{0}, T\right), \mathbf{a}\right)=\min _{t_{0} \leq t \leq T} v_{E}^{(t)}\left(\mathfrak{R}\left(t_{0}, T\right), \mathbf{a}\right) ;
$$

-minimal angular velocity of scenario $\mathfrak{R}\left(t_{0}, T\right)$ with regard to vector $\mathbf{a}$

$$
v_{a}^{(\min )}\left(\mathfrak{R}\left(t_{0}, T\right), \mathbf{a}\right)=\min _{t_{0} \leq t \leq T} v_{a}^{(t)}\left(\mathfrak{R}\left(t_{0}, T\right), \mathbf{a}\right) ;
$$

-maximal velocity of scenario $\mathfrak{R}\left(t_{0}, T\right)$ in the direction of vector $\mathbf{a}$

$$
v_{E}^{(\min )}\left(\mathfrak{R}\left(t_{0}, T\right), \mathbf{a}\right)=\max _{t_{0} \leq t \leq T} v_{E}^{(t)}\left(\Re\left(t_{0}, T\right), \mathbf{a}\right) ;
$$

-maximal angular velocity of scenario $\mathfrak{R}\left(t_{0}, T\right)$ with regard to vector $\mathbf{a}$

$$
v_{a}^{(\min )}\left(\mathfrak{R}\left(t_{0}, T\right), \mathbf{a}\right)=\max _{t_{0} \leq t \leq T} v_{a}^{(t)}\left(\mathfrak{R}\left(t_{0}, T\right), \mathbf{a}\right) .
$$

Let us set vector $\mathbf{a} \in Z$. Assume that in the expanded phase space on the horizon $T$ there are set scenarios $\mathfrak{R}_{1}$ of SES development with the initial ESE $\mathfrak{J}_{e v}^{\left(\alpha_{1}(0)\right)}\left(t_{0}\right)=\left\{Z^{\left(\alpha_{1}(0)\right)}, \mathbf{z}^{(1)}\left(t_{0}\right), t_{0}\right\}$ and scenarios $\mathfrak{R}_{2}$ with the initial ESE $\mathfrak{I}_{e v}^{\left(\alpha_{2}(0)\right)}\left(t_{0}\right)=\left\{Z^{\left(\alpha_{2}(0)\right)}, \mathbf{z}^{(2)}\left(t_{0}\right), t_{0}\right\}$, constructed according to rules $0-0$. 
Definition 1. Scenario $\mathfrak{R}_{1}$ will be called a scenario of outstripping development in the direction of vector a on the horizon $T$ as compared to scenario $\mathfrak{R}_{2}$, if

$$
\rho_{a}\left(\mathbf{z}^{(1)}(t), \mathbf{a}\right) \leq \rho_{a}\left(\mathbf{z}^{(2)}(t), \mathbf{a}\right) \text { for all } t \in[0, T] .
$$

Vector $\mathbf{a}$ is a vector of the desirable direction of SES development. Condition (11) means that on the horizon $T$ scenario $\mathfrak{R}_{1}$ is closer to vector $\mathbf{a}$ as compared to scenario $\mathfrak{R}_{2}$, with regard to the angular distance. Definition 1 enables scenario comparison of by their closeness to the specified direction of SES development in the expanded phase space $Z$.

Let us assme that

$\Delta\left(\rho_{a}, \mathbf{a}, \mathfrak{R}_{1}, \mathfrak{R}_{2}, t\right)=\rho_{a}\left(\mathbf{z}^{(1)}(t), \mathbf{a}\right)-\rho_{a}\left(\mathbf{z}^{(2)}(t), \mathbf{a}\right)$,

i.e. the angular mismatch between the scenarios with regard to the direction $\mathbf{a}$ at time $t$.

Definition 2. Scenario $\mathfrak{R}_{1}$ will be called a scenario of overtaking development in the direction of vector on the horizon $T$ with growth rate $\delta$, as compared to scenario $\mathfrak{R}_{2}$, iffor $\delta>0$ the following holds:

$$
\Delta\left(\rho_{a}, \mathbf{a}, \mathfrak{R}_{1}, \mathfrak{R}_{2}, t\right) \geq \delta \text { for all } t \in[0, T]
$$

Definition 2 makes it possible to compare scenarios by the rate of their approach to the set development of SES in the expanded phase state $Z$.

Let us assign a set of characteristics as functionals from $M \mathfrak{J}_{e v}$ in $E^{S}$

$$
\Omega \mathbf{f}\left(\mathfrak{I}_{e v}\right)=\left\{f_{\tau}\left(\mathfrak{I}_{e v}\right), \tau=\overline{1, S}\right\} \subseteq E^{S}(14)
$$

of events significant to the expert $\mathfrak{I}_{e v} \in M \mathfrak{I}_{e v}$, as well as a set of subsets

$$
\begin{gathered}
\bar{M}^{\Re} \mathbf{f}\left(\mathfrak{I}_{e v}\right)=\left\{\bar{M}^{\Re} f_{\tau}\left(\mathfrak{I}_{e v}\right), \tau=\overline{1, S}\right\} \subseteq E^{S}, \\
\breve{M}^{\Re} \mathbf{f}\left(\mathfrak{J}_{e v}\right)=\left\{\breve{M}^{\Re} f_{\tau}\left(\mathfrak{I}_{e v}\right), \tau=\overline{1, S}\right\} \subseteq E^{S}
\end{gathered}
$$

interpreted as permissible or desirable values of characteristics $\mathbf{f}$.

Definition 3. An expert significant event $\mathfrak{J}_{e v}$ is called permissible with regard to characteristic $f_{\tau}$ in scenario $\mathfrak{R}$, or $f_{\tau}$-permissible if

$$
f_{\tau} \in \widehat{M}^{\Re} f_{\tau}\left(\Im_{e v}\right) .
$$

An expert significant event $\mathfrak{I}_{\text {ev }}$ is called desirable with regard to characteristic $f_{\tau}$ in scenario $\mathfrak{R}$, or $f_{\tau}$ desirable, if

$$
f_{\tau} \in \breve{M}^{\Re} f_{\tau}\left(\mathfrak{J}_{e v}\right)
$$

Denote a set of $f_{\tau}$-permissible ESE as $\widehat{M}_{e v}^{\Re} f_{\tau} \subseteq \mathfrak{I}_{e v} Z T$. We will call an ESE $\mathfrak{J}_{e v}$, permissible by all characteristics $f_{\tau} \tau=\overline{1, S}, \mathbf{f}$-permissible for scenario $\mathfrak{R}$.

Denote a set of $\mathbf{f}$-permissible for scenario $\mathfrak{R}$ ESE as $\widehat{M}_{e v}^{\Re} \mathbf{f}$.

Denote a set of $f_{\tau}$-desirable ESE as $\breve{M}_{e v}^{\Re} f_{\tau} \subseteq \mathfrak{I}_{e v} Z T$. An ESE $\mathfrak{J}_{e v}$, permissible by all characteristics $f_{\tau}$, will be called $\mathbf{f}$-desirable for scenario $\mathfrak{R}$.

Let us denote a set of $\mathbf{f}$ - desirable for scenario $\mathfrak{R}$ ESE as $\breve{M}_{e v}^{\Re} \mathbf{f}$.

Example 1. Permissible and prioritative ESE.

Let there be assigned a set of conditionally permissible states $\hat{Q}$, i.e.

$$
\mathbf{z}(t) \in \operatorname{int} \hat{Q} .
$$

Expression 0 models necessary conditions of SES development trajectory membership in the set $\widehat{Q}$.

Let there be set an ESE 0. Let us introduce a Boolean characteristic

$$
q\left(\mathfrak{J}_{e v}, \hat{Q}\right)=\left\{\begin{array}{l}
0, \text { if } \mathbf{z}(t) \in \operatorname{int} \hat{Q} \\
1, \text { if } \mathbf{z}(t) \notin \operatorname{int} \hat{Q}
\end{array} .\right.
$$

Then conditions 0 can be written in the language 0 as follows:

$$
q\left(\mathfrak{I}_{e v}, \widehat{Q}\right)=0,
$$

i.e. ESE $\mathfrak{J}_{e v}^{\alpha}(t)=\left\{Z^{\alpha}, \mathbf{z}(t), t\right\}$ is $q$-permissible for scenario $\mathfrak{R}$ if 0 holds.

Let a set of prioritative states $\breve{R}$ be given, that is condition

$$
\mathbf{z}(t) \in \breve{R}
$$

is the most desirable to the decision maker.

Assume an ESE 0 is assigned. Let us introduce a Boolean characteristic 


$$
r\left(\mathfrak{\Im}_{e v}, \breve{R}\right)=\left\{\begin{array}{l}
0, \text { if } \mathbf{z}(t) \in \breve{R} \\
1, \text { if } \mathbf{z}(t) \notin \breve{R}
\end{array} .\right.
$$

Then conditions 0 could be written using the language 0 as

$$
r\left(\mathfrak{J}_{e v}, \breve{R}\right)=0,
$$

i.e. ESE $\mathfrak{J}_{e v}^{\alpha}(t)=\left\{Z^{\alpha}, \mathbf{z}(t), t\right\}$ is $r$-permissible for scenario $\mathfrak{R}$ if 0 holds.

Definition 3 enables determination of optimal with regard to criterion 0 (prioritative) and permissible by criterion 0 ESE as ESE out of the set

$$
\mathfrak{J}_{e v} \in M_{e v}^{\Re}(q, r)=M_{e v}^{\Re} q \cap M_{e v}^{\Re} r .
$$

Such ESE $\mathfrak{J}_{e v}$ are permissible and optimal for scenario $\Re$.

Definition 3 and Example 1 help generalise definitions of ESE optimality and permissibility in the case when an ESE 0 does not belong to set (24), i.e. the ESE is either nonpermissible or non-prioritative for scenario $\mathfrak{R}$.

Example 2. Generally permissible and optimal ESE.

Let us set numbers $\varepsilon$ and $\delta$ and introduce characteristics of ESE 0 in space $Z$

$$
q\left(\mathfrak{J}_{e v}, \widehat{Q}\right)=\rho_{Z}(\mathbf{z}, \widehat{Q})=\inf _{\mathbf{v} \in \hat{Q}} \rho_{Z}(\mathbf{z}, \mathbf{v}),
$$

i.e. the distance to the set of conditionally permissible states and

$$
r\left(\mathfrak{J}_{e v}, \breve{R}\right)=\rho_{Z}(\mathbf{z}, \breve{R})=\inf _{\mathbf{v} \in \bar{R}} \rho_{Z}(\mathbf{z}, \mathbf{v}),
$$

i.e. the distance to the set of conditionally prioritative states and

Definition 4. We will consider that ESE $\mathfrak{I}_{e v}$ is $\delta$. permissible if

$$
q\left(\mathfrak{I}_{e v}, \hat{Q}\right) \leq \delta
$$

i.e. the distance from $\mathfrak{I}_{e v}$ to set $\widehat{Q}$ does not exceed $\delta$.

Definition 5. We will call ESE $\mathfrak{J}_{e v} \quad \varepsilon$-optimal if

$$
r\left(\mathfrak{I}_{e v}, \breve{R}\right) \leq \varepsilon,
$$

which means that the distance from $\mathfrak{J}_{e v}$ to the set of prioritative states does not exceed $\varepsilon$.

Thus a 0-permissible ESE is contained in the set of conditionally permissible states but a 0 -optimal ESE is contained in the set of prioritative states in the case if these sets are closed.
Definitions of QIH, strategies and characteristics of a scenario allow one to formally state the task of observation and analysis of the process of factor transformation as well as its representation as a totality of $M \mathfrak{R}^{(s y n)}$ synergetic scenarios each of which indicates a fundamental way of SES development.

Assume there is assigned a collection 0 , as well as a set $\bar{M}^{\Re} \mathbf{f}$ of their permissible values.

Task 1. To accomplish a synthesis of the synergetic scenario $\mathfrak{R}^{(s y n)}$, that is on the assigned horizon of the scenario, $t=\left[t_{0}, t_{1}\right]$ to show a sequence of conditions

$$
\mathfrak{R}^{(\text {syn })}=\mathfrak{R}^{(\text {syn })}\left\{\left(I^{(s y n)}(t), t\right)\right\},
$$

where $t=\overline{0, T}, \mathfrak{R}^{(s y n)} \in M \mathfrak{R}^{(s y n)}$,

but $I^{(s y n)}(t)$ - conditions at time instant $t$ for which a collection of characteristics 0 is $\mathbf{f}$-permissible.

Determination of controls, resources and rules for choosing effective strategies of ESE formation makes it possible to state tasks of control of the process of parameter transformation and its representation in the form of an attractive scenario $\mathfrak{R}^{\text {(atr) }}$.

Task 2.To construct an attractive scenario $\mathfrak{R}^{(\text {opt })}$, that is to carry out the synthesis of optimal control in the form of a sequence of $\mathbf{f}$-permissible ESE

$$
\mathfrak{R}^{(\text {atr })}=\mathfrak{R}^{(\text {atr })}\left\{\left(I^{(\text {atr })}(t), t\right)\right\} \text {, where } t=\overline{0, T} \text {. }
$$

where $t_{0}=\min _{s \in g} t_{0}^{(s)}, t_{1}=\max _{s \in g} t_{1}^{(s)}$.

A scenario of that kind is formed according to this rule:

$$
\mathfrak{R}^{(\text {atr })}=\mathfrak{R}^{(\text {atr })}\left\{\left(I^{(\text {atr })}(t), t\right)\right\} \text {, где } t=\overline{0, T} .
$$

where $I^{(a t r)}(t)$ - conditions at time instant $t$. In contrast to the synergetic scenario, the procedure of control formation is its obligatory element.

\section{CONCLUSIONS}

The proposed statement of the task of synthesis of synergetic and attractive scenarios allows one to consider tasks of that kind in a general aspect for different subject areas, different employed models describing the behaviour of the object and its environment as well as for different kinds of uncertainty accounted within the scenario-based research of SES. 


\section{REFERENCES}

Kononov D.A., Kul'ba V.V., Malugin V.D., Shubin A.N. 2005. Information processes and information control. Proceedings „Human Factors in Management”, SINTEG, Moscow. [In Russian].

Kononov D.A., 2007. Synthesis of Social Economic System Development Scenarios with Operator graph Apparatus. Avtom. Telemekh., No. 1. [in Russian]

Roberts, F.S., 1976. Discrete Mathematical Models with Application to Social, Biological, and Enviromental Problems. New York: Prentice Hall, 1976.

Kul'ba V.V., Kononov D.A., Kosjachenko S.A., Shubin A.N., 2004. Design Techniques of Development Scenarios of Social Economic Systems. SINTEG, Moscow. [In Russian].

Kononov D.A., 2001. Effective Scenarios Synthesis Strategies in Automatic Control Systemems in Emergency Situation. Avtom. Telemekh., No. 2, pp, 170-181. [in Russian]

Novikov D.A., Chartishvili, 2003. Reflexive games. SINTEG, Moscow. [In Russian].

\section{AUTHOR BIOGRAPHIES}

VLADIMIR V. KUL'BA, Academician, Dr.habil.sc., Professor, Trapeznikov Institute of Control Sciences Russian Academy of Sciences. He has made significant contribution in areas of control theory, optimal modular data processing, local and distributed data bases, network computing systems, and others.

DMITRY A. KONONOV, Dr.Math., Professor, Trapeznikov Institute of Control Sciences, Russian Academy of Sciences, Moscow, Russia. His research area is development of mathematical models and methods for analysis and synthesis of complex systems' scenario.

GALINA MERKURYEVA, DSc., Dr.sc.ing., Professor at the Department of Modelling and Simulation, Riga Technical University, Latvia. Research interests are in the fields of discrete-event computer simulation and metamodelling methodology, knowledge-based simulation systems, simulation-based training and fuzzy systems theory. 